Citation: E. Pacetti, A. Soriani (2021) Pratiche mediali, social media e influencers nella formazione e nell'espressione identitaria dei bambini: una ricerca esplorativa. Media Education 12(2): 35-46. doi: 10.36253/me-11406

Received: June, 2021

Accepted: October, 2021

Published: December, 2021

Copyright: (C) 2021 E. Pacetti, A. Soriani. This is an open access, peer-reviewed article published by Firenze University Press (http://www.fupress.com/me) and distributed under the terms of the Creative Commons Attribution License, which permits unrestricted use, distribution, and reproduction in any medium, provided the original author and source are credited.

Data Availability Statement: All relevant data are within the paper and its Supporting Information files.

Competing Interests: The Author(s) declare(s) no conflict of interest.

\section{Pratiche mediali, social media e influencers nella formazione e nell'espressione identitaria dei bambini: una ricerca esplorativa ${ }^{1}$}

\author{
Digital media practices, social media and influencers in \\ children's identity formation and expression: an exploratory \\ research.
}

\author{
Elena Pacetti, Alessandro Soriani \\ Dipartimento di Scienze dell'Educazione, Università di Bologna \\ elena.pacetti@unibo.it; alessandro.soriani@unibo.it
}

\begin{abstract}
With the spread of social media, we witnessed the multiplication of web's figures such as influencers and content creators. The pupils in the last years of primary schools (9-11 years old), engaged in a complex task of defining their own identity, are strongly exposed to entertainment contents conveyed by social networks and online video platforms. The present exploratory study, which involved 173 students from a primary school in Emilia-Romagna Region (Italy), aims to investigate the role of social media and influencers in the formation and expression of the identities of children, highlighting a universe little known and only superficially explored by teachers and adults with educational roles. The contribution has the twofold objective of: investigating children's practices related to social networks (in particular those based on video content such as YouTube, Twitch, TikTok, Instagram and Snapchat) to offer an overview of the situation in an age group increasingly targeted by these social networks; making a reflection on how much the figures of content creators and influencers play a role in the identity formation of children in $4^{\text {th }}$ and $5^{\text {th }}$ year of primary school. The results lead to new research questions and educational path to make teachers and parents more aware of this issue.
\end{abstract}

Keyword: influencers, social media, primary school, identity.

Riassunto. Contestualmente al diffondersi delle piattaforme social, si è assistito alla moltiplicazione, sul web, di figure quali influencers o content creators. Gli studenti frequentanti gli ultimi anni di scuola primaria, impegnati in un complesso compito di definizione della propria identità, sono fortemente esposti a contenuti di intrattenimento veicolati da social e piattaforme video online. La presente ricerca, un'indagine

\footnotetext{
${ }^{1}$ Il presente contributo può essere attribuito come segue: i paragrafi 1, 2, 3, 8, 9 sono stati scritti da Alessandro Soriani; i paragrafi 4, 5, 6, 7, 10 sono stati scritti da Elena Pacetti. Si desidera inoltre ringraziare Marina Cembali, studentessa in Scienze della Formazione Primaria, per il lavoro svolto nella raccolta dati.
} 
esplorativa che ha coinvolto 173 studenti di una scuola primaria dell'Emilia-Romagna, ha avuto l'obiettivo di indagare il ruolo dei social media e degli influencers nella formazione e nellespressione identitaria dei giovani in età preadolescenziale, mettendo in risalto un universo poco conosciuto e solo superficialmente esplorato dagli insegnanti e dagli adulti con ruoli educativi. Il contributo si pone il duplice obiettivo di: indagare le pratiche relative ai social network (in particolare quelli basati sui contenuti video come YouTube, Twitch, TikTok e Instagram) dei giovani alunni e delle giovani alunne al fine di offrire una panoramica della situazione in una fascia d'età sempre più targhettizzata da questi social; compiere una riflessione su quanto la figura dell'influencer giochi un ruolo nella formazione identitaria dei bambini e delle bambine. I risultati portano a nuove domande di ricerca e a percorsi formativi per consapevolizzare maggiormente insegnanti e genitori su questo tema.

Parole chiave: influencers, social media, scuola primaria, identità.

\section{SEMPRE PIÜ CONNESSI: LE PRATICHE DEI BAMBINI}

Ben prima dell'emergenza Covid-19, situazione che ha forzato milioni di bambini e ragazzi a comunicare in maniera quasi del tutto esclusiva attraverso tecnologie digitali, l'utilizzo di smartphone, tablet e altri dispositivi per connettersi alla rete era già in fortissimo incremento (Istat, 2019; 2020).

Secondo il report EU Kids Online 2020: Survey results from 19 countries (Smahel et al., 2020) in Italia prevale un utilizzo di smartphone e di dispositivi mobile per connettersi alla rete: i bambini e ragazzi dai 9 ai 16 anni accedono ad internet prevalentemente da smartphone o altri dispositivi mobile (80\%), mentre il $39 \%$ vi accede da computer portatili, laptop o desktop e solo il $20 \%$ da tablet. Consultando il report Europeo si può avere un'idea più precisa del tempo trascorso online dai giovani italiani: si parla di una media di 143,5 minuti al giorno per i giovani da 9 a 16 anni e, più nello specifico, di 93 minuti per i bambini e le bambine dai 9 agli 11 anni. Le attività preferite dai giovani italiani sono riassunte nella tabella sottostante e messe a confronto con i valori medi in Europa: guardare videoclip online (1'82\% ha risposto giornalmente, più di una volta al giorno o sempre), comunicare con famiglia e amici (74\%), visitare social network (54\%), ascoltare musica online (47\%), usare internet per la scuola (35\%) e giocare a videogiochi online e $28 \%$ ).

Durante la pandemia e la condizione di isolamento che milioni di bambini e giovani preadolescenti sono stati costretti a vivere, è stato registrato in tutto il mondo un sensibile aumento del tempo trascorso online (Sultana et al., 2021). Secondo una ricerca comparativa tra Italia e Francia svolta nel 2020, che in Italia ha riguardato un campione di 4.477 bambini e ragazzi minori di 16 anni, è stato evidenziato che durante il lockdown gli studenti in età di scuola primaria hanno raddoppiato il tempo passato guardando la televisione o altri schermi in modo passivo: in Italia l'incremento è stato da 1,5 ore a 3 ore di media al giorno, mentre in Francia da 1 a 2 ore di media giornaliera (Champeaux et al., 2020).

Nel resto del mondo il trend non è dissimile: secondo il rapporto annuale di Common Sense Media (Rideout \& Robb, 2019), in America, nel 2019 - dati pre-covid quindi - il tempo medio gionnaliero di utilizzo di media dotati di schermo da parte dei giovani in età fra 8 e 12 anni, era di 4,44 ore. Questo non includendo le ore davanti allo schermo passate a scuola o a casa facendo i compiti.

Se si osserva la situazione nel Regno Unito (OFCOM, 2020), si può notare che oltre la metà dei ragazzi di 10 anni possiede un proprio smartphone personale e che il consumo di Video-on-demand (attraverso piattaforme come YouTube, Netflix o Twitch) è incrementato sensibilmente (salendo dal 44\% nel 2015 all' $80 \%$ nel 2019). I dispositivi preferiti per accedere a tali contenuti sono TV smart (91\% di utenti nel 2019, percentuale in calo rispetto al 2015 che contava il 96\%), Tablet (percentuale che è salita dal 27\% nel 2015 al 43\% del 2019) e Smartphone (in crescita anch'essa: 15\% nel 2015 e $26 \%$ nel 2019). Per quanto riguarda il solo uso di YouTube, le percentuali rispetto all'uso della piattaforma da parte di ragazzi dagli 8 agli 11 anni è del 74\% e dell'89\% per ragazzi dai 12 ai 15 anni.

Un ulteriore elemento da non trascurare all'interno delle pratiche mediali giovanili è il fenomeno del

Tabella 1. Pratiche giovanili secondo il rapporto EU Kids Online 2020.

\begin{tabular}{lcc}
\hline & $\begin{array}{c}\text { Valore medio in } \\
\text { Italia }\end{array}$ & $\begin{array}{c}\text { Valore medio in } \\
\text { Europa }\end{array}$ \\
\hline Guardare videoclip online & 82 & 65 \\
Comunicare con famiglia e amici & 74 & 61 \\
Visitare social network & 54 & 54 \\
Ascoltare musica online & 47 & 65 \\
Usare internet per la scuola & 35 & 31 \\
Giocare a videogiochi online & 28 & 44
\end{tabular}


gaming. Tale pratica ricreativa era già interessata da una continua e costante crescita, su scala globale, in termini di ore di gioco e di giocatori coinvolti, ma in seguito alla pandemia tale trend ha visto un ulteriormente incremento. Secondo il rapporto OFCOM (2020), nel Regno Unito, il 59\% dei bambini e dei ragazzi dai 5 ai 15 anni gioca online (nel 2015 la percentuale era del "solo" 45\%). Secondo il rapporto ISFE per l'anno 2020 (2021) il 73\% dei bambini da 6 a 10 anni e 1'84\% dei ragazzi dagli 11 ai 14 anni hanno giocato a videogiochi. A livello italiano (IIDEA, 2021), invece, nel 2020 i giovani dai 6 ai 14 anni rappresentano il $21 \%$ dell'intera popolazione di videogiocatori che si attesta intorno ai 16,7 milioni di italiani (il 38\%).

Quello del gaming è un elemento da tenere fortemente in considerazione dato che è possibile giocare su praticamente ogni dispositivo digitale: computer, portatili, tablet, console da gioco fisse o portatili e, naturalmente, smartphone. I dispositivi più utilizzati dai giocatori italiani fra i 6 e i 10 anni sono console da gioco (44\%) e smartphone (36\%) (ibidem) mentre tra gli 11 e i 14 anni si assiste ad un'inversione della tendenza vedendo i dispositivi mobile come device preferiti (62\%), seguiti dalle home consoles (56\%). L'interesse così forte da parte dei ragazzi e delle ragazze verso questa pratica non si esprime solamente attraverso la semplice azione del gioco, ma è accompagnata da tutta una serie di altre pratiche ad esso collegate che li vedono impegnati in una fittissima rete di relazioni sociali mediate sia dai giochi stessi (attraverso il multiplayer, specialmente quello online), sia dai canali di comunicazione digitale, sia da alcune piattaforme social (YouTube e Twitch in primis) dove si costituiscono community partecipatissime e dove si assiste a complessi e diversificati fenomeni di socializzazione ai media (Caronia, 2002). Nel momento in cui si scrive questo articolo (28 giugno 2021), infatti, fra i dieci canali di YouTube con più visite in Italia, quattro sono a tema videoludico: WhenGamersFail > Lyon, FavijTV, St3pNy e Two Players One Console ${ }^{2}$.

\section{RUOLO DEI SOCIAL E DELLE COMUNICAZIONI MEDIATE DA TECNOLOGIE}

Le tecnologie e i media sono entrati a far parte della nostra quotidianità in una maniera ormai irreversibile tanto da rendere impossibile concepire un mondo in cui offline ed online sussistano in maniera distinta e separata: sono essi stessi, i media, ad essere andati onlife (Floridi, 2017) modificando ogni aspetto della nostra società.

\footnotetext{
${ }^{2}$ https://socialblade.com/youtube/top/country/it/mostviewed (ultimo
} accesso: 28/06/2021)
Questa cornice è importante per comprendere meglio come i giovani possono capire e dare senso a quello che stanno vivendo.

Ormai iperconnessi (Twenge, 2018), in costante bilico fra una presenza reale e una virtuale, i pre-adolescenti di oggi sono osservati con molta preoccupazione da parte del mondo adulto, il quale spesso li considera veri e propri "sdraiati" digitali ${ }^{3}$. Certamente i rischi legati all'abuso di tecnologie sono tanti e difficili da gestire: abuso di smartphone, dipendenza da internet e da videogiochi, fear of missing out, sexting, cyberbullying, grooming, rappresentano problemi reali sui quali bisogna confrontarsi e intorno ai quali costruire un dialogo aperto, sincero e positivo con i ragazzi (Manca, 2016).

A tal scopo, si possono identificare due aspetti da tenere in considerazione. In primo luogo, il percorso evolutivo che i ragazzi e le ragazze sono impegnati ad intraprendere e che li vede immersi in un'importante serie di cambiamenti sia fisiologici (dovuti alla pubertà) che contestuali (cambio di scuola, ampliamento del bacino di pari con cui relazionarsi). In questo delicato momento il compito principale è quello di formare la propria identità in un complesso negoziato fra prospettiva personale, confronto con pari e con adulti (Côté \& Levine, 2016; Erikson, 1968). Le tecnologie ricoprono, all'interno di questo processo, un ruolo fondamentale poiché diventano un terreno di incontro con i propri pari - libero da molti vincoli spazio-temporali - altro. Uno spazio in cui poter sperimentare identità (boyd, 2014) e agire relazioni (Turkle, 1995, 2012). Uno spazio di cui difficilmente si riesce a fare a meno perché risulta un costante flusso di scambi relazionali che diventa prassi quotidiana e risposta ad uno dei bisogni fondamentali per un ragazzo o una ragazza in quell'età: il bisogno di contatto con i pari. Le ricerche in questo campo sono numerose e affrontano il tema dell'adolescenza e dei social media da prospettive differenti: ora più incentrate sullo sviluppo evolutivo degli adolescenti, esaminandone anche le pratiche principali, (Manca, 2016), ora focalizzandosi sulle dinamiche emozionali ed affettive (Scarcelli, 2015), ora, ancora, osservando le influenze dei social media sulle dinamiche di evoluzione identitaria negli adolescenti (Lim, 2015; Bissaca, Cerulo \& Scarcelli, 2020).

Il secondo elemento da non sottovalutare, che si intreccia a doppio filo con quanto appena illustrato, riguarda l'aspetto consumistico legato all'uso delle tecnologie. Gardner \& Davis (2014) hanno osservato come l'utilizzo di app in maniera così massiva non solo influenzi molti aspetti legati all'identità di una persona,

${ }^{3}$ Il termine nasce parafrasando Michele Serra e il suo libro Gli sdraiati, edito nel 2013 da Feltrinelli. 
ma che promuova la formazione di un senso del sé preconfezionato, omologato. Oltre questo rischio è importante considerare anche la natura fortemente consumistica, di stampo neoliberista, del mercato dell'industria hi-tech (Buckingham, 2020; Potter \& McDougall, 2017). Le grosse compagnie leader del settore (Facebook, Amazon, Google - ovvero Alphabet - ed Apple) non sono semplicemente enti filantropici che regalano la possibilità di avere una casella e-mail o di comunicare attraverso messaggi coi propri amici, ma sono colossi industriali che mirano a fatturare, a creare nuovi clienti e a fidelizzare quelli già posseduti anche attraverso sistemi partecipativi basati su internet celebrities (Abidin, 2018). Questa 'spinta' non può essere ignorata se si vuole osservare in maniera complessa e problematica la motivazione per cui i giovani sono attratti dalle tecnologie.

\section{IL RUOLO DEGLI INFLUENCERS}

Il termine 'influencer' è nato e si è sviluppato nell'ambito del marketing online. Agli albori del web 2.0, una ricerca condotta dall'Università di Rutgers (Bickart \& Schindler, 2001) dimostrò come sempre più utenti consultassero forum online per cercare consigli da fonti autorevoli su articoli da acquistare. A partire da quel momento, sempre più aziende iniziarono ad implementare - attraverso mailing list organizzate o veri e propri portali come BlogStar ${ }^{4}$ o PayPerPost ${ }^{5}$ - sistemi per chiedere agli utenti più autorevoli di forum o di blog di promuovere (o semplicemente parlare) di particolari prodotti commerciali dietro un pagamento ${ }^{6}$. Da quel momento in poi, con l'avvento e la diffusione di social network quali YouTube, Facebook, Instagram e Twitter, il fenomeno è letteralmente esploso assumendo tratti sempre più di difficile delineamento e coinvolgendo fasce d'età più vicine al mondo degli adolescenti e dei preadolescenti.

Limitarsi però a considerare il campo di influenza degli influencers come legato esclusivamente al dominio del marketing sarebbe semplicistico e riduttivo. Jerslev (2016) offre una definizione del termine influencer descrivendo coloro che appartengono a questa categoria come soggetti che usano le loro abilità comunicative per influenzare il comportamento e le opinioni di terzi. Freberg et al. (2011), e Lou \& Yuan (2019) ampliano questa

\footnotetext{
${ }^{4}$ https://blogstar.co.uk/ (ultimo accesso: 28/06/2021)

${ }^{5} \mathrm{https}: / /$ payperpost.com/ (ultimo accesso: 28/06/2021)

${ }^{6}$ Per avere una prospettiva storica sul fenomeno Influencer, si rimanda all'articolo di Martineau (2019) su Wired online, reperibile a questo indirizzo: https://www.wired.com/story/what-is-an-influencer/ (ultimo accesso: 28/06/2021)
}

definizione considerando gli influencers sui social media come content creators esperti in un particolare campo di competenza o più (la cultura nerd, i videogiochi, le ricette, la moda, etc.) in grado di modellare i comportamenti, le scelte e le decisioni riguardo ad acquisti dei propri followers.

È proprio questo il punto chiave: comportamenti, opinioni e scelte costituiscono una parte fondamentale del percorso di negoziazione identitaria dei ragazzi e delle ragazze in età preadolescenziale e adolescenziale; età nella quale si trovano in una fase molto delicata del loro sviluppo che li rende fortemente influenzabili e sensibili a quello che vedono intorno a loro, tecnologie e media digitali compresi (Bernete, 2009).

Le pratiche degli adolescenti legate a piattaforme di video online, YouTube in primis, sono fortemente correlate con una serie di bisogni che Lenhart e colleghi (2015) identificano nei seguenti: bisogno di collaborazione con i propri pari e con i familiari; bisogno di interazione con altri spettatori; bisogno di coinvolgimento in dinamiche di cittadinanza attiva; formazione della propria identità. Oltre a queste quattro non bisogna dimenticare la posizione di studiosi come Katz, Gurevitch e Haas (1973), padri della teoria degli usi e delle gratificazioni, i quali identificano cinque bisogni che sono soddisfatti dai media:

- bisogni cognitivi, inteso come acquisizione e rafforzamento di conoscenze e saperi;

- bisogni affettivi ed estetici, inteso come gestione delle emozioni e sensibilizzazione all'esperienza esteti$\mathrm{ca}$;

- bisogni integrativi legati allo sviluppo e alla definizione della personalità;

- bisogni integrativi sociali ed interpersonali;

- bisogni di evasione, inteso come sfogo di tensioni o come esperienze ricreative.

Le figura dei content creator e degli influencer, diventano quindi vettore di intrattenimento e, contemporaneamente, simbolo di un divenire 'possibile'. Questo è reso possibile da due elementi. In primis, l'autenticità di queste 'micro celebrità', che valorizzano un modo di fare percepito come più autentico, meno artefatto, più sincero (Aran-Ramspott, et al, 2018). In secondo luogo, il senso di vicinanza e di possibile/plausibile immagine di sé che gli influencers trasmettono, divenendo di fatto role-model molto vicini all'idea di successo che desiderano i ragazzi: successo percepito come facilmente o comunque realisticamente emulabile (Lou \& Kim, 2019) nonstante una certa lontananza dalle reali possibilità.

In conclusione a questa prima cornice si può affermare che le ricerche che esplorano lo sviluppo identitario dei giovani in relazione alle loro pratiche mediatiche, 
come è emerso anche dai dati esposti, sembrano essere prevalentemente focalizzate sul target pre-adolescenziale e adolescenziale e meno ad un target in età da primaria. Con la presente ricerca ci si propone, a fronte di un largo utilizzo delle tecnologie digitali da parte di bambini e bambine dai 9 agli 11 anni, di analizzare la complessità del fenomeno cercando di comprendere nello specifico quali sono le pratiche relative ai social network degli studenti frequentanti gli ultimi anni di scuola primaria e quanto gli influencers presenti sui vari social giochino un ruolo nella formazione delle loro identità.

\section{OBIETTIVO E DOMANDE DELLA RICERCA}

La presente ricerca, dal carattere prevalentemente esplorativo, è stata progettata al fine di mettere in luce le pratiche mediali relative ai social media dei bambini negli ultimi anni di primaria (9-11 anni) e di fornire ai loro insegnanti una panoramica, seppur ristretta, della problematica, in modo che possano meglio comprenderne la portata. Alla luce dell'analisi svolta, arricchita da riflessioni raccolte durante questo particolare anno di emergenza didattica, lo studio si propone di mettere in evidenza quanto tali pratiche giochino un ruolo chiave, nella formazione e nell'espressione delle identità dei bambini e delle bambine tra i 9 e gli 11 anni.

In particolare, l'indagine si focalizza sulle seguenti domande di ricerca:

- Quali sono le pratiche relative ai social network (in particolare quelli basati sui contenuti video) di alunni ed alunne frequentanti il $4^{\circ}$ e $5^{\circ}$ anno di scuola primaria?

- Secondo quali modalità i content creator e gli influencer presenti sui social influenzano l'espressione identitaria di bambini e bambine del $4^{\circ}$ e $5^{\circ}$ anno di scuola primaria?

\section{NOTE METODOLOGICHE}

La ricerca, di tipo esplorativo, è stata condotta durante l'anno scolastico 2019/2020 presso una scuola primaria appartenente ad un Istituto Comprensivo di Lugo di Romagna. Sono state coinvolte tutte le classi quarte e quinte dell'IC per un totale di otto classi: quattro quarte e quattro quinte. La scelta di limitare la distribuzione dei questionari a studenti degli ultimi due anni di primaria è motivata prevalentemente da due motivi: coinvolgere studenti di un'età intorno ai 9-11 anni ha permesso, primo, di ottenere un bacino di utenti certamente più vicini alle pratiche social e, secondo, di essere più sicuri circa la comprensione e la compilazione del questionario.

Sono stati distribuiti 180 questionari cartacei agli studenti delle otto classi e ne sono stati raccolti 173 (85 studenti di classe quarta e 88 di quinta per un totale di 99 studenti e 74 studentesse). I questionari, compilabili in circa 30-40 minuti, erano composti da tre sezioni:

- la prima, finalizzata a raccogliere le informazioni base sui partecipanti alla ricerca come dati anagrafici o possesso di strumenti digitali;

- la seconda, relativa a indagare le pratiche relative a social network degli studenti e delle studentesse;

- la terza, specificamente ideata al fine di comprendere il ruolo delle star di YouTube e di altri influencers nella vita di alunne e alunni delle classi coinvolte.

I questionari erano composti da domande chiuse, per rilevare o meno la presenza di pratiche relative all'uso dei social, e aperte, aventi invece lo scopo di cogliere le motivazioni e le argomentazioni delle risposte chiuse. Al fine di favorire la compilazione dei questionari da parte delle classi campione, la studentessa Marina Cembali è rimasta, durante la somministrazione, in aula a disposizione di tutti gli alunni ed alunne supportando anche i casi di alunni con disabilità (1 presente) o disturbo specifico di apprendimento (2 presenti).

Le risposte alle domande chiuse sono state raccolte in un foglio elettronico e poi trattate attraverso SPSS, un software di analisi quantitativa che ha permesso la costruzione di grafici e tabelle. Un'ultima precisazione: il questionario conteneva come possibili social network incentrati su condivisione di video e di immagini, YouTube, TikTok, Instagram, Twitch e la possibilità di inserire altri social utilizzati non elencati. Solamente Snapchat è stato aggiunto alla lista, pertanto figura nei grafici a fianco degli altri.

Le domande aperte sono state analizzate e codificate in modo da fare emergere direttamente dai dati una categorizzazione delle stesse (Charmaz, 2006).

Visto il numero di studenti coinvolti, la ricerca non ha avuto l'intento di comprendere il fenomeno degli influencers nella sua interezza, ma piuttosto quello di tracciare un profilo delle pratiche adottate da un campione ristretto allo scopo di conoscere meglio la problematica e di sollevare una serie di riflessioni utili agli insegnanti per avere più contezza delle pratiche dei loro alunni relative al mondo degli influencers.

\section{POSSESSO DEI DISPOSITIVI}

Prima di procedere presentando gli usi dei social network da parte dei bambini, è interessante soffermarsi 
sui dati che raccontano il possesso o l'accesso all'uso dei dispositivi digitali più utilizzati. Smartphone, console da gioco e tablet sono i dispositivi più diffusi. Fra questi, lo strumento più popolare è certamente lo smartphone: il $43,3 \%$ ne possiede uno personale e il $33 \%$ ha accesso ad uno smartphone in condivisione con i genitori o altre persone del nucleo familiare.

Le console da gioco sono molto diffuse, a testimoniare il fatto che la pratica del videogioco è davvero molto comune: infatti, oltre la metà (il 52,5\%) possiede una console da gioco personale, mente il 19,7\% ha risposto di averne una in casa, ma di non esserne il diretto proprietario. Il 45,9\% degli studenti e delle studentesse coinvolte ha risposto di avere un tablet personale e il $26,2 \%$ di averne uno a disposizione a casa non personale. I dispositivi più "tradizionali" risultano invece meno diffusi come strumenti personali: solo il $16,40 \%$ possiede un laptop suo e appena il 9,3\% un computer fisso personale. Computer fisso e portatile risultano essere però gli strumenti più utilizzati in condivisione con altri membri della famiglia con il 38,3\% della presenza di un laptop non personale e il $41,5 \%$ per il PC.

\section{PRATICHE RELATIVE AI SOCIAL MEDIA}

Nei grafici sottostanti sono raccolte le risposte relative alle domande "Con che frequenza fruisci di queste piattaforme?" e "Per quanto tempo al giorno utilizzi queste piattaforme?".

Per quanto concerne l'utilizzo delle piattaforme social, YouTube spicca come piattaforma più utilizzata, seguita da TikTok e Instagram. Gli stessi trend sono riscontrabili osservando i dati relativi all'utilizzo delle piattaforme da parte degli alunni maschi e delle alunne femmine, dove però emergono una serie di differenze, seppur minori, che vale la pena sottolineare:

- YouTube tende ad essere uno strumento utilizzato più frequentemente da maschi;

- Twitch, seppur ancora poco utilizzato in generale, è più utilizzato da un'audience maschile;

- TikTok ed Instagram sono piattaforme più utilizzate da un target femminile.

Guardando al tempo giornaliero passato sulle piattaforme social identificate, dichiarato da alunni ed alunne, emerge un andamento non dissimile dagli usi presentati nel grafico 1 e nella tabella 2. YouTube è la piattaforma su cui alunne ed alunni passano più tempo, seguito da TikTok e da Instagram. Di particolare interesse è l'attenzione riposta dal pubblico femminile nella piattaforma cinese: il $17,3 \%$ delle ragazze, infatti, ha dichiarato di passare più di due ore al giorno su TikTok. Le alunne parte della ricerca hanno espresso un utilizzo di TikTok molto più frequente e molto più continuativo nella giornata rispetto ai maschi, i quali, invece riportano di apprezzare maggiormente contenuti video provenienti da YouTube e da Twitch.

Per quanto riguarda le pratiche degli studenti e delle studentesse relative ai diversi social network si può affermare che YouTube rimane la piattaforma più utilizzata in forma passiva (cioè da semplice fruitore) anche se risulta fra quelle meno utilizzate secondo un profilo d'uso più attivo: il $94,5 \%$ segue diversi canali, il $30,1 \%$ ha un profilo utente registrato e solo il $5,5 \%$ possiede un canale proprio. Instagram, invece, risulta essere la seconda piattaforma più fruita in maniera passiva ma quella più utilizzata attivamente: ben il 18\% dei partecipanti alla ricerca afferma di avere un profilo proprio che utilizza per caricare contenuti, questo con una netta prevalenza delle

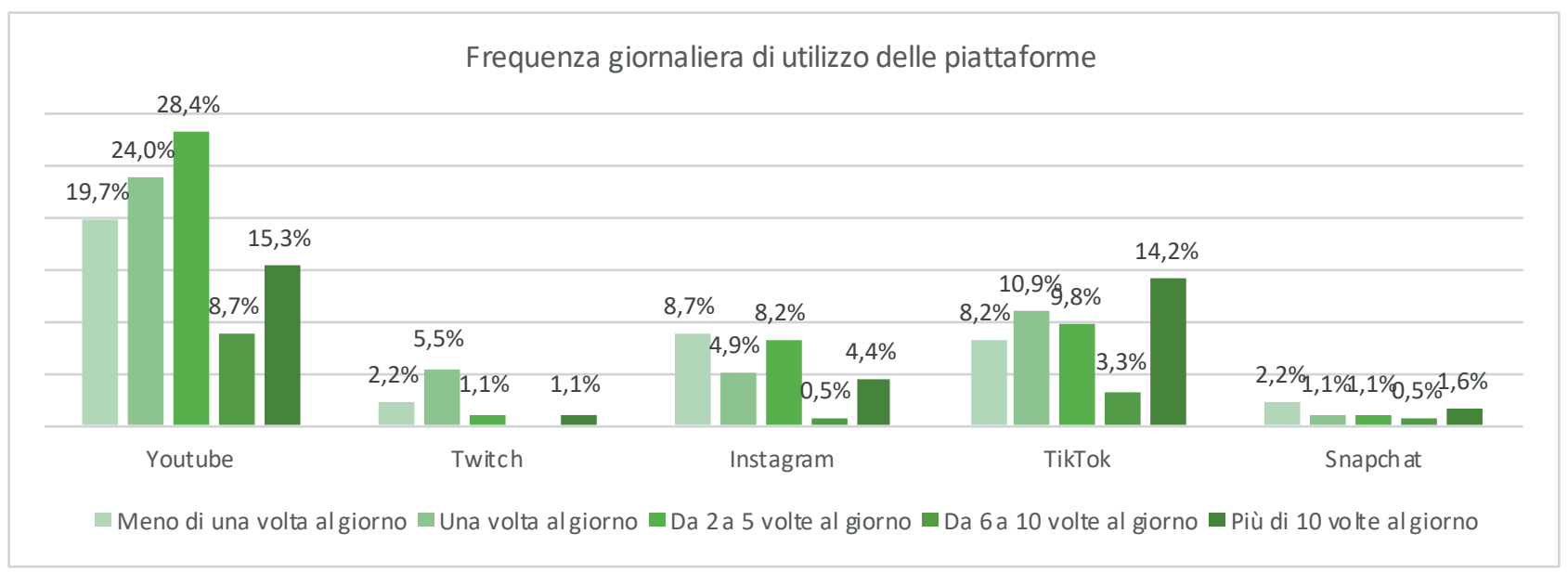

Grafico 1. Utilizzo delle piattaforme social (\%). 
Tabella 2. Utilizzo delle piattaforme social organizzate per genere (\%).

\begin{tabular}{|c|c|c|c|c|c|c|c|c|c|c|}
\hline & \multicolumn{2}{|c|}{ Meno di 1/gg } & \multicolumn{2}{|c|}{$1 / \mathrm{gg}$} & \multicolumn{2}{|c|}{$2-5 / g g$} & \multicolumn{2}{|c|}{$6-10 / g g$} & \multicolumn{2}{|c|}{ Più di 10/gg } \\
\hline & M & $\mathrm{F}$ & M & $\mathrm{F}$ & M & $\mathrm{F}$ & M & F & M & $\mathrm{F}$ \\
\hline YouTube & $16,7 \%$ & $24,0 \%$ & $23,1 \%$ & $25,3 \%$ & $28,7 \%$ & $28,0 \%$ & $5,6 \%$ & $13,3 \%$ & $19,4 \%$ & $9,3 \%$ \\
\hline TikTok & $3,7 \%$ & $0,0 \%$ & $7,4 \%$ & $2,7 \%$ & $1,9 \%$ & $0,0 \%$ & $0,0 \%$ & $0,0 \%$ & $1,9 \%$ & $0,0 \%$ \\
\hline Instagram & $6,5 \%$ & $12,0 \%$ & $0,9 \%$ & $10,7 \%$ & $9,3 \%$ & $6,7 \%$ & $0,0 \%$ & $1,3 \%$ & $3,7 \%$ & $5,3 \%$ \\
\hline Twitch & $2,8 \%$ & $16,0 \%$ & $10,2 \%$ & $12,0 \%$ & $10,2 \%$ & $9,3 \%$ & $3,7 \%$ & $2,7 \%$ & $10,2 \%$ & $20,0 \%$ \\
\hline Snapchat & $1,9 \%$ & $2,7 \%$ & $0,9 \%$ & $1,3 \%$ & $0,0 \%$ & $2,7 \%$ & $0,9 \%$ & $0,0 \%$ & $0,0 \%$ & $4,0 \%$ \\
\hline
\end{tabular}

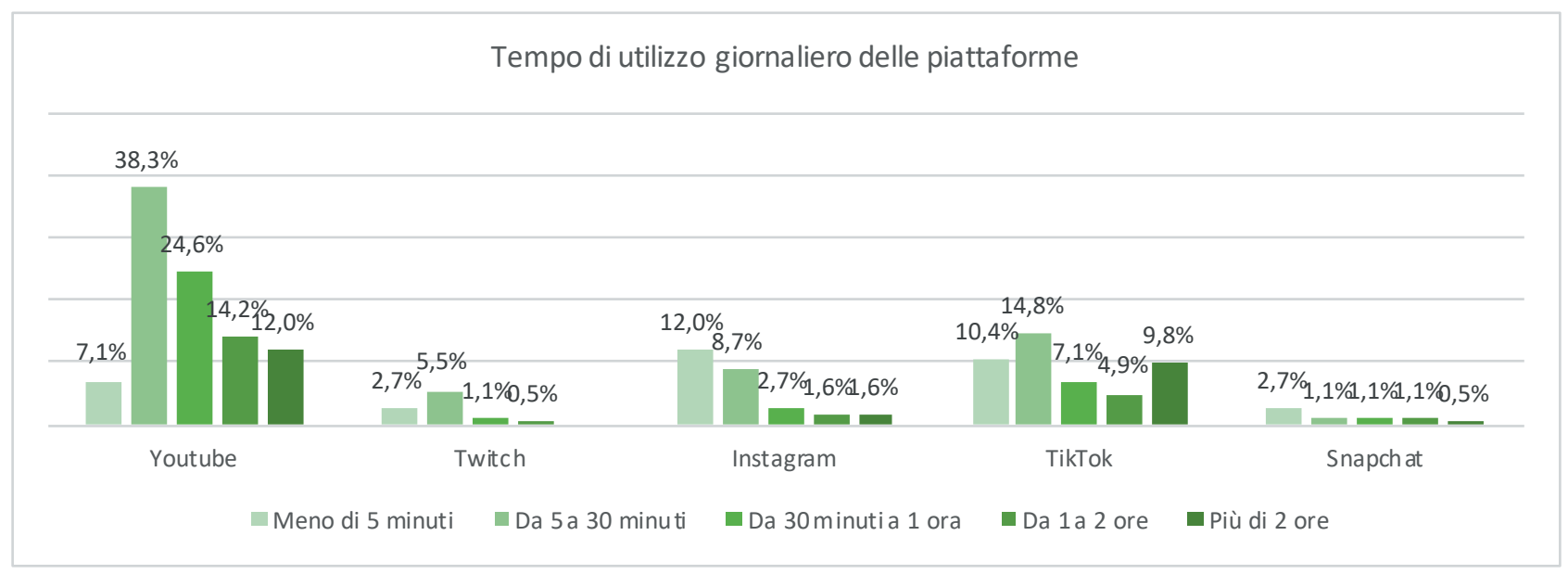

Grafico 2. Tempo giornaliero passato sulle piattaforme social (\%).

Tabella 3. Tempo giornaliero passato sulle piattaforme social per genere (\%).

\begin{tabular}{lcccccccccc}
\hline & $<5 \min (\mathrm{M})<5 \min (\mathrm{F})$ & $\begin{array}{c}5-30 \mathrm{~min} \\
(\mathrm{M})\end{array}$ & $\begin{array}{c}5-30 \mathrm{~min} \\
(\mathrm{~F})\end{array}$ & $\begin{array}{c}30 \mathrm{~min}-1 \mathrm{~h} \\
(\mathrm{M})\end{array}$ & $\begin{array}{c}30 \mathrm{~min}-1 \mathrm{~h} \\
(\mathrm{~F})\end{array}$ & $1-2 \mathrm{~h}(\mathrm{M})$ & $1-2 \mathrm{~h}(\mathrm{~F})$ & $>2 \mathrm{~h}(\mathrm{M})$ & $>2 \mathrm{~h}(\mathrm{~F})$ \\
\hline Youtube & $6,5 \%$ & $8,0 \%$ & $38,0 \%$ & $38,7 \%$ & $23,1 \%$ & $26,7 \%$ & $12,0 \%$ & $17,3 \%$ & $13,9 \%$ & $9,3 \%$ \\
Twitch & $3,7 \%$ & $1,3 \%$ & $9,3 \%$ & $0,0 \%$ & $0,9 \%$ & $1,3 \%$ & $0,9 \%$ & $0,0 \%$ & $0,0 \%$ & $0,0 \%$ \\
Instagram & $8,3 \%$ & $17,3 \%$ & $6,5 \%$ & $12,0 \%$ & $2,8 \%$ & $2,7 \%$ & $1,9 \%$ & $1,3 \%$ & $0,9 \%$ & $2,7 \%$ \\
TikTok & $8,3 \%$ & $13,3 \%$ & $12,0 \%$ & $18,7 \%$ & $7,4 \%$ & $6,7 \%$ & $4,6 \%$ & $5,3 \%$ & $4,6 \%$ & $17,3 \%$ \\
Snapchat & $1,9 \%$ & $4,0 \%$ & $0,0 \%$ & $2,7 \%$ & $0,9 \%$ & $1,3 \%$ & $0,0 \%$ & $2,7 \%$ & $0,9 \%$ & $0,0 \%$ \\
\hline
\end{tabular}

bambine $(26,7 \%)$ rispetto ai bambini (12\%). Interessante notare, infine, come TikTok si attesti come seconda piattaforma più utilizzata in maniera attiva con un $8,2 \%$ di alunni ed alunne.

Risultano particolarmente interessanti le risposte alle domande aperte relative ai tipi di contenuti più seguiti sui social. Gli argomenti dei canali YouTube più seguiti da bambini e bambine sono: videogiochi (85 risposte), challenge o video divertenti (59), tutorial vari (2), sport (1) e vlog personali (15). Per quanto riguarda gli argomenti dei canali creati dagli studenti troviamo: videogiochi (6), vlog personali (5). Le risposte relative ai contenuti più seguiti su TikTok, invece, sono: balletti musicali (40 risposte), tutti i video che capitano (23), challenge o video divertenti (20). I canali TikTok creati invece dagli alunni riguardano prevalentemente balletti musicali (18) e challenge o video divertenti (10). Si noti come la risposta "guardo tutti i video che capitano" sia particolarmente calzante per il social network in esame, il quale si presenta come un infinito flusso di contenuti video in grado di catturare l'attenzione per minuti ed ore intere (Lupinacci, 2020). 


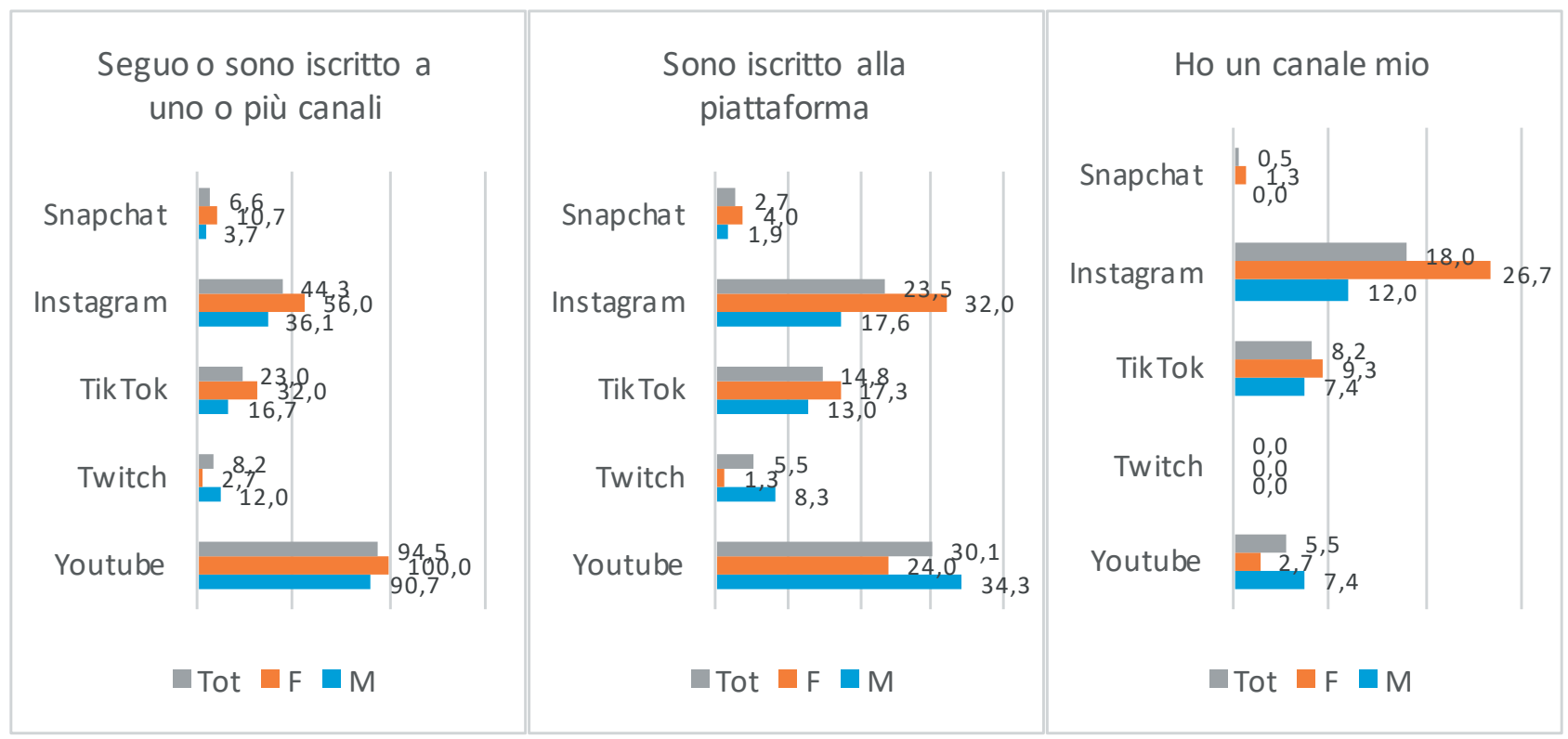

Grafico 3. Pratiche relative alle differenti piattaforme (\%).

\section{INFLUENCERS}

Dai risultati del questionario risulta che ben oltre la metà dei bambini e bambine che hanno aperto un canale YouTube o un profilo TikTok l'abbiano fatto ispirandosi ad uno o più influencers già esistenti; infatti, del 23,5\% degli studenti che possiede un profilo sul quale carica contenuti (43 risposte), ben il 64,5\% di questi (26) ha dichiarato di essersi ispirato a canali di YouTuber o di TikToker già esistenti. Com'è possibile osservare dal grafico sottostante, non vi sono particolari divergenze fra le risposte degli alunni e quelle delle alunne.

Le risposte raccolte rivelano la presenza di una forte carica emulativa che alcuni content creators esercitano sui più piccoli. Tale considerazione è corroborata anche dai dati che emergono dalle risposte alle domande aperte rela-

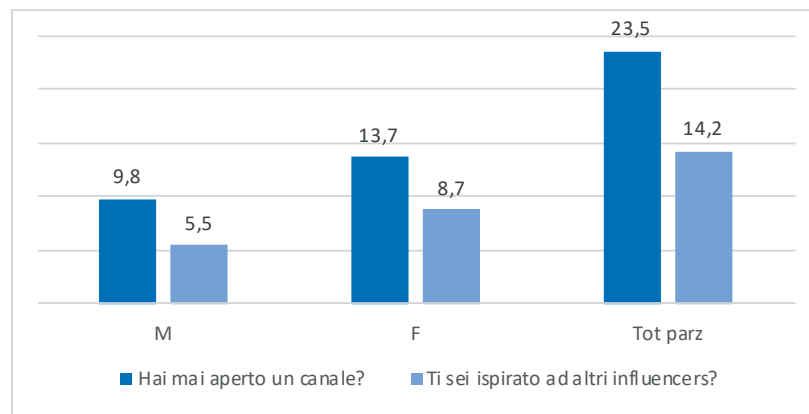

Grafico 4. Apertura di canali YouTube o TikTok e fonte di ispirazione (\%). tive al ruolo degli influencers in diversi elementi della vita degli alunni (grafico 5): in primis il processo di acquisto di libri, videogiochi, o di visione di film o serie tv; in secondo luogo il processo di cambio di opinione su qualcosa in seguito alla visione di un video online; terzo, il processo di ispirazione alle star del web nel vestire o nel modo di tenere i capelli; ed infine l'utilizzo di modi di dire o gerghi sentiti online da YouTuber o altri content creators.

\section{Acquisti e consigli}

Questa categoria di influenza risulta la più rilevante, con ben il $45 \%$ degli studenti che ha affermato di essersi lasciato guidare da suggerimenti lanciati da content creators, online, nell'acquisto di un oggetto o di aver iniziato a fruire di contenuti video (film o serie Tv). Ecco alcuni numeri: 28 studenti hanno dichiarato di aver seguito questi consigli per la visione di film, 13 per la visione di serie Tv, e 7 per la visione di altri canali YouTube; 16 soggetti hanno affermato di aver comprato libri di YouTuber, 9 altri oggetti (giocattoli, e gadget vari) e 6 altri libri non scritti da YouTuber.

\section{Modi di dire}

La seconda categoria per ordine di risposte è quella relativa ai modi di parlare e di dire che i bambini e le bambine hanno fatto propri dopo averli sentiti dalle star del web che seguono: il $38,25 \%$ ha risposto che utilizza 


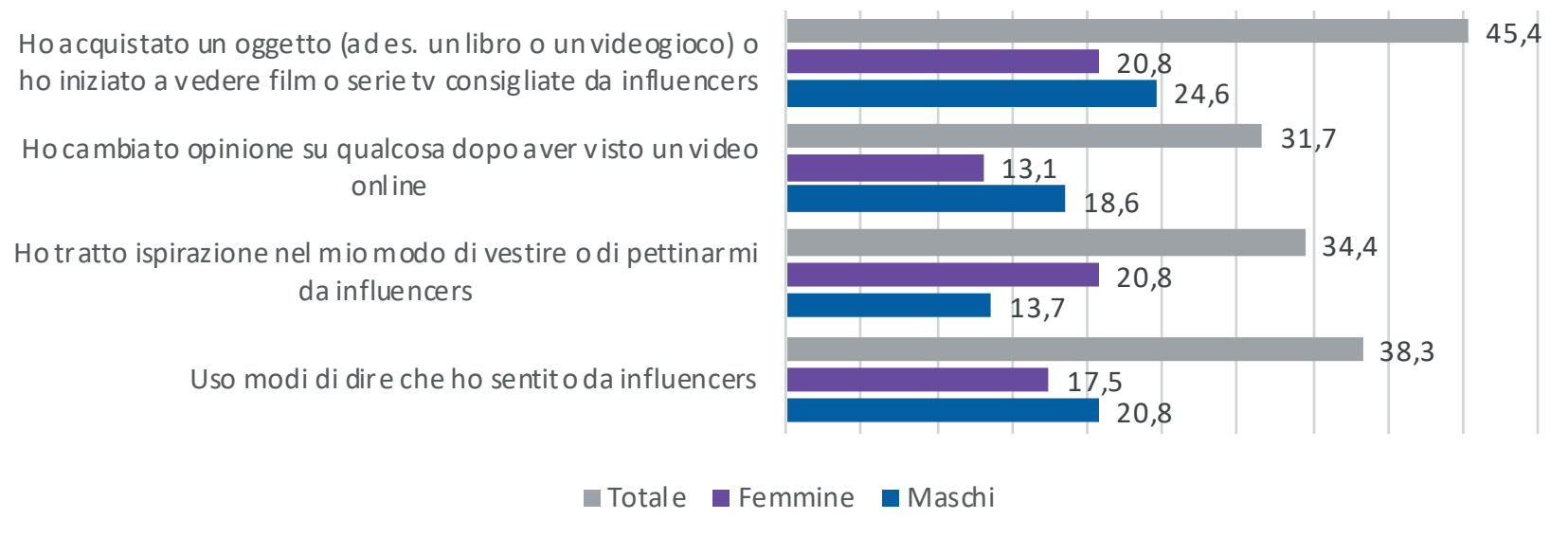

Grafico 5. Ruolo degli influencers nelle scelte personali (\%).

abitualmente esclamazioni gergali (15), modi di dire ed intercalari (14), maniere di salutare (10), altre espressioni e costrutti di frasi (8) e modi di dire provenienti da Meme (5) che hanno sentito online.

\section{Abbigliamento e capelli}

Il $34,4 \%$ degli alunni e delle alunne ha risposto di trarre ispirazione da alcuni influencers nel modo di vestire o di portare i capelli. In particolare, la categoria di influencers più seguita, in questo senso, sono gli YouTuber (20), seguita da content creators di TikTok (9) e Instagram (7). Solo 5 alunni hanno affermato di ispirarsi a personaggi di film o serie Tv.

\section{Cambiamenti di opinione rispetto a vari argomenti}

Il 31,7\% dei rispondenti al questionario ha ammesso di aver cambiato, in seguito alla visione di contenuti online su piattaforme come YouTube, TikTok o Instagram, la propria opinione su alcuni elementi. 13 alunni/e hanno risposto di aver modificato i propri comportamenti ("Quando penso che una cosa sia vera, ma alla fine no", "Di avere ottimismo invece che vedere negativo", "Sul modo di parlare alle persone"); 9 hanno risposto di aver cambiato opinione su uno o più videogiochi; 6 riguardo a fatti di attualità; 3 di aver cambiato opinione sul loro modo di vestire e 2 sulla loro alimentazione.

\section{CONCLUSIONI}

La ricerca, pur se dal carattere principalmente esplorativo e pur se condotta su un campione ristretto di alunni/e, ha confermato l'utilizzo dello smartphone quale strumento maggiormente diffuso già fra bambini di quarta e di quinta primaria. Inoltre, ha posto in evidenza come l'utilizzo dei social media sia molto diffuso già dagli ultimi anni della scuola primaria e come YouTuber e TikToker siano diventati i principali punti di riferimento delle giovani generazioni. Anche se la maggior parte di ragazzi e ragazze usufruisce in maniera passiva dei contenuti social (da spettatore che segue e osserva), alcuni di loro hanno profili attivi e producono propri contenuti sui principali canali a loro disposizione (Instagram, TikTok, Snapchat e YouTube), in parte con il desiderio di emulare chi è già diventato famoso su quei canali e in parte con il desiderio (questo molto più presente) di allargare la propria rete di contatti e di "pubblici" (boyd, 2014). Le loro abitudini e comportamenti sono influenzati, sia nelle scelte di consumo, sia nei modi di pensare, di affrontare le situazioni, di osservare il mondo intorno a loro non solo da quanto vedono pubblicato online, ma anche dal tipo di relazione para sociale che instaurano con i propri influencers simile ad una amicizia, ovviamente solo percepita come già evidenziato da altre ricerche sugli adolescenti (Lou \& Kim, 2019).

Gli studenti degli ultimi anni di primaria sono una categoria che fa parte a pieno titolo del mercato presente in rete, non solo come consumatori, ma anche come gruppo attivo che, ugualmente, esprime preferenze e gusti, riflettendo sulle proprie pratiche e formandosi in contesti informali. Prevale, infatti, un utilizzo spontaneo e uniforme dei nuovi media, capace di rendere i giovani followers parte dello stesso gruppo, così come accade per gruppi di adolescenti (Fornasari, 2017): utilizzare le stesse parole e frasi, vestirsi in un certo modo, fare gli stessi balletti consente a bambine e bambini di condividere modelli e contribuisce a formare la propria identità 
in un'epoca post-mediale in cui "I media sono ovunque. Noi stessi siamo i media” (Eugeni, 2015, p.416). Ma, lo ricordiamo, è un'epoca in cui le pratiche mediali vengono vissute solo fra i pari, senza alcuna mediazione del mondo adulto e senza la presenza di famiglie e insegnanti nei contesti non formali e formali.

In questo senso, le famiglie appaiono scarsamente preparate a questo scenario: in Italia, infatti, emerge non solo un quadro in cui le competenze digitali sono ancora a livelli piuttosto bassi (Istat, 2019) - ma anche una evidente difficoltà, da parte dei genitori, di gestire ed accompagnare i bambini nel loro rapportarsi ed utilizzare le tecnologie. Le famiglie con meno grado di istruzione sono quelle che concedono lo smartphone ai propri figli già a partire dai 9 anni (Gui et al., 2020) e, pur essendo utilizzate anche in famiglia per mantenere i legami, solo nel 53\% delle famiglie avviene una conversazione quotidiana riguardante le regole sul loro consumo, mentre esiste una percentuale importante (37\%) di genitori che non si preoccupa di ciò che i figli pubblicano online (CIRF, 2017). I più giovani, quindi, sono esposti ai media digitali fin da piccoli, ma senza la necessaria mediazione da parte delle famiglie e senza essere adeguatamente formati a un loro utilizzo critico ed etico (Guerra, 2020). Se da una parte le famiglie non sembrano adeguatamente preparate ad accompagnare $i$ propri figli nell'utilizzo dei nuovi media, anche il mondo della scuola fatica a introdurre la media education nella quotidianità didattica. La ricerca EU Kids online aveva già evidenziato nel 2017 che gli insegnanti italiani prediligono una mediazione restrittiva all'uso di Internet (regole, controllo e proibizioni) piuttosto che una mediazione attiva (incoraggiare ad esplorare e acquisire competenze) (Mascheroni \& Ólafsson, 2018). Come dire che è più semplice proibire che educare a un utilizzo critico e consapevole. Ma certamente questo dato riflette le difficoltà che molti insegnanti hanno nell'uso delle tecnologie: "Mentre in molti Paesi OCSE gli insegnanti utilizzano le TIC con pari intensità rispetto ad altri lavoratori con istruzione terziaria, gli insegnanti italiani rimangono indietro e utilizzano le nuove tecnologie ben al di sotto di altri lavoratori altamente qualificati. I dati mostrano, inoltre, come 3 insegnanti su 4 riferiscano di aver bisogno di ulteriore formazione nelle TIC per svolgere la propria professione" (Rapporto OCSE, 2019, p.2). Anche dalla ricerca TALIS 2018 (OECD, 2019), pur essendo rivolta ai soli insegnanti di scuola secondaria di primo grado, emerge che gli insegnanti italiani che si sentono preparati all'uso delle tecnologie dell'informazione applicato alla didattica rappresentano il 35,6\% (media OCSE 42,8\%) e che il 16,6\% dichiara un elevato livello di necessità di sviluppo professionale nell'ambi- to delle competenze nell'uso didattico delle TIC (media OCSE 17,7\%).

E dunque, in particolare, la scuola a risultare in una situazione di maggior bisogno: a fronte di cospicui investimenti che il MIUR ha attuato nel paese, l'utilizzo delle tecnologie non è diffuso in maniera omogenea, creando situazioni molto diversificate (Proietti et al., 2017) e questa situazione di precarietà e di lontananza dall'uso delle ICT è emersa maggiormente durante l'emergenza Covid-19 e il lockdown delle scuole nel 2020 e 2021, che ha costretto le scuole ad adottare la Didattica a distanza o la Didattica Integrata. La ricerca promossa da Indire (2020) conferma un diffuso bisogno di formazione su aspetti tecnologici e sull'uso di piattaforme/strumenti digitali (ad esempio, nel $33 \%$ dei docenti della primaria). Sono inoltre i docenti che già promuovono una didattica innovativa e laboratoriale a dimostrarsi ancora più propensi ad ulteriori formazioni in futuro (rispetto a docenti più tradizionali). Il Censis (2020) ha rilevato un'inadeguatezza degli istituti scolastici di fronte all'emergenza del coronavirus: nelle interviste svolte ad aprile 2020, 1'82,1\% dei 2.812 dirigenti scolastici ha affermato che gli ostacoli principale per docenti e studenti sono stati sia le differenti dotazioni tecnologiche, sia la diversa familiarità d'uso.

La ricerca esplorativa apre, dunque, diverse ulteriori prospettive di indagine: in primo luogo, il fenomeno andrebbe indagato a livello nazionale su un campione più ampio per analizzare le pratiche mediali di bambine e bambini negli ultimi anni della scuola primaria e verificare se tali pratiche siano diffuse e in che modo in Italia. In secondo luogo, l'indagine andrebbe estesa a famiglie e insegnanti per comprendere il loro punto di vista e la loro consapevolezza sull'uso che i propri figli/ alunni fanno dei social media e quanto questi influenzino la loro formazione identitaria. Se i dati analizzati si confermassero anche a livello nazionale, si evidenzierebbe ancor di più l'urgenza di educare i più piccoli a un utilizzo critico e consapevole dei social media.

Diventa allora fondamentale, sempre di più, che la scuola sia in grado di accompagnare i propri alunni nell'acquisizione di competenze digitali che rispondano a un diritto di cittadinanza nella società digitale. Fin dai primi anni di scuola, alunne e alunni devono poter praticare la media education, un'educazione con i media, ai media e per i media (Rivoltella, 2015) per formare cittadini critici, responsabili e attivi che siano non solo consumatori e riproduttori di stereotipi, ma anche produttori di cultura e contenuti originali, con competenza. "L'agire autonomo e responsabile delle persone competenti conferisce al concetto di competenza un significato non solo cognitivo, pratico, metacognitivo, ma anche e soprattutto etico" (MIUR, 2018, p.5). Una cittadinan- 
za digitale, dunque, che promuova anche l'uso dei social media per affrontare tematiche non solo di intrattenimento, ma anche politiche e di impegno civile. "Uno spazio come YouTube, dunque, in cui potenzialmente ciascuno può essere sé stesso e parlare di questioni che lo riguardano direttamente, rappresenta un potente mezzo di contaminazione culturale e un ambiente inclusivo reale, in cui i soggetti, nel rispetto delle loro peculiarità, hanno la possibilità di mettere in discussione i modelli culturali e i rapporti di forza dominanti, ma soprattutto di autogestire consapevolmente il proprio Sé" (Bocci, De Castro, Zona, 2020).

\section{RIFERIMENTI BIBLIGRAFICI}

Abidin, C. (2018). Internet Celebrity: Understanding Fame Online. Bingley: Emerald Group Pub Ltd.

Aran-Ramspott, S., Fedele, M., \& Tarragó, A. (2018). YouTubers' social functions and their influence on pre-adolescence. Comunicar, 57, 71-80. https://doi. org/10.3916/C57-2018-07

Beyens, I., Pouwels, J. L., van Driel, I. I. et al. (2020) The effect of social media on well-being differs from adolescent to adolescent. Sci Rep, 10, 10763. https://doi. org/10.1038/s41598-020-67727-7

Bernete, F. (2009). Usos de las TIC, relaciones sociales y cambios en la socialización de los jóvenes. Revista de Estudios de Juventud. 88, 97-114. Retrieved from http://www.injuve.es/sites/default/files/RJ88-08.pdf

Bickart, B., Schindler, R. M. (2001). Internet Forums As Influential Sources Of Consumer Information. In Journal of Interactive Marketing. 15 (3). 31-40. http:// dx.doi.org/10.1002/dir.1014

Bissaca, E., Cerulo, M., Scarcelli, C. M. (2020). Giovani e social network. Emozioni, costruzione dell'identità e media digitali. Roma: Carrocci Editore.

Bocci, F., De Castro, M., Zona, U. (2020). Non solo marketing. Lecosistema YouTube come opportunità per l'autonarrazione della disabilità e dell'inclusione. In MeTis. Mondi educativi. Temi, indagini, suggestioni, v.10, 1, 121-138. https://10.30557/MT00115

boyd, danah. (2014). It's Complicated: The Social Lives of Networked Teens. New Heaven and London: Yale University Press.

Buckingham, D. (2020). Un manifesto per la media education (Trad. Ital.). Milano: Mondadori Università.

Caronia, L. (2002). La socializzazione ai media. Contesti, interazioni e pratiche educative. Milano: Guerini e Associati.

Censis (2020). $54^{\circ}$ rapporto sulla situazione sociale del paese 2020. Milano: FrancoAngeli.
Champeaux, H., Mangiavacchi, L., Marchetta, F., Piccoli, L. (2020). Learning at Home: Distance Learning Solutions and Child Development during the COVID-19 Lockdown. In IZA Discussion Papers, 13819. Retrieved from: https://www.econstor.eu/handle/10419/227346

Charmaz, K. (2006). Constructing Grounded Theory. London: SAGE Publications.

CIRF Centro Internazionale Studi Famiglia (2017) (a cura di). Le relazioni familiari nell'era delle reti digitali. Cinisello Balsamo: Edizioni San Paolo.

Côté, J. E., \& Levine, C. G. (2016). Identity Formation, Youth, and Development. New York: Psychology Press.

Erikson, E. H. (1968). Identity: youth and crisis. New York: W. W. Norton Company.

Eugeni, R. (2015). La condizione Postmediale: media, linguaggi e narrazioni. Brescia: La Scuola.

Fornasari, A. (2017). Social Privacy. Informare, comunicare, educare ai tempi del web 3.0. Mondo Digitale, 16(71), 1-13. Retrieved from http://mondodigitale. aicanet.net/2017-4/articoli/MD71_FORNASARI_ ARTICOLO_.pdf

Floridi, L. (2017). La quarta rivoluzione. Come l'infosfera sta trasformando il mondo. Milano: Raffaello Cortina.

Freberg, K., Graham, K., McGaughey, K., \& Freberg, L. A. (2011). Who are the social media influencers? A study of public perceptions of personality. Public Relat. Rev. 37, 90-92. https://10.1016/j.pubrev.2010.11.001

Gardner, H., \& Davis, K. (2014). The App Generation: How Today's Youth Navigate Identity, Intimacy, and Imagination in a Digital World. Yale University Press.

Guerra, M. (2020). Una scuola autentica in un mondo virtuale. MeTis. Mondi educativi. Temi, indagini, suggestioni, 10, 1, 197-209. Retrieved from http://www. metisjournal.it/index.php/metis/article/view/368/279

Gui, M., Gerosa, T., Vitullo, A., Losi, L. (2020). Letà dello smartphone. Unianalisi dei predittori sociali dell'età di accesso al primo smartphone personale e delle sue possibili conseguenze nel tempo. Report del Centro di ricerca Benessere Digitale. Università di Milano Bicocca. Retrieved from http://www.benesseredigitale.ue/ pubblicazioni

IIDEA. (2021). I videogiochi in Italia nel 2020. Dati sul mercato e sui consumi. Milano: IIDEA, Italian Interactive Digital Entertainment Association. Retrieved from https://iideassociation.com/kdocs/2003252/ iidea_i_videogiochi_in_italia_nel_2020.pdf

Indire (2020). Indagine tra $i$ docenti italiani. Pratiche didattiche durante il lockdown. Report integrativo, dicembre 2020. Retrieved from https://www.indire. 
it/wp-content/uploads/2020/12/Report-integrativoNovembre-2020_con-grafici-1.pdf

ISFE. (2021). Key Facts 2020. Brussels: Europe's Video Games Industry. Retrieved from https://www.isfe.eu/ wp-content/uploads/2020/08/ISFE-final-1.pdf

Istat (2019). Cittadini e ICT. Anno 2019. Retrieved from https://www.istat.it/it/archivio/236920

Istat (2020). Spazi in casa e disponibilità di computer per bambini e ragazzi. Retrieved from https://www.istat. it/it/files/2020/04/Spazi-casa-disponibilita-computerragazzi.pdf

Jerslev, A. (2016). In the time of the microcelebrity: Celebrification and the youtuber Zoella. International Journal of Communication, 10, 5233-5251. Retrieved from https://bit.ly/2shZq4T

Katz, E., Gurevitch, M., \& Haas, H. (1973). On the Use of the Mass Media for Important Things. American Sociological Review, 38 (2), 164-181.

Lenhart, A., Smith, A., Anderson, M., Duggan, M., \& Perrin, A. (2015). Teens, technology and friendships. Pew Research Center. Retrieved from https://www.pewresearch.org/2015/08/06/teens-technology-and-friendships/

Lim, S.S. (2015). Media and peer culture: Young people sharing norms and collective identities with and through media. In D. Lemish (Ed.), The Routledge International Handbook of Children, Adolescents and Media. London: Routledge.

Lou, C., \& Yuan, S. (2019). Influencer marketing: how message value and credibility affect consumer trust of branded content on social media. In Journal of Interactive Advertising, 19, 58-73. https://doi.org/10.1080/ 15252019.2018 .1533501

Lou, C., \& Kim, H. K. (2019). Fancying the New Rich and Famous? Explicating the Roles of Influencer Content, Credibility, and Parental Mediation in Adolescents' Parasocial Relationship, Materialism, and Purchase Intentions. Frontiers in Psychology. 10, 2567. https://doi.org/10.3389/fpsyg.2019.02567

Lupinacci, L. (2020). "Absentmindedly scrolling through nothing": liveness and compulsory continuous connectedness in social media. In Media, Culture \& Society. https://doi.org/10.1177/0163443720939454

Manca, M. (2016). Generazione Hashtag. Roma: Alpes Edizioni

Mascheroni, G. \& Ólafsson, K. (2018). Accesso, usi, rischi e opportunità di internet per i ragazzi italiani. I risultati di EU Kids Online 2017. Retrieved from http:// globalkidsonline.net/wp-content/uploads/2017/10/EUKids-Online-Italy-report-06-2018.pdf

MIUR (2018). Indicazioni Nazionali e nuovi scenari. Retrieved from https://www.miur.gov.it/docu-
ments/20182/0/Indicazioni+nazionali+e+nuovi+sce nari/

OCSE (2019). Skills Outlook Scoreboard. Prosperare in un mondo digitale. Retrieved from https:// d1 10 erj1750600.cloudfront.net/wp-content/ uploads/2019/05/Skills-Outlook-Italy-IT.pdf

OECD (2019). TALIS 2018 Results (Volume I): Teachers and School Leaders as Lifelong Learners. Paris: OECD Publishing.

OFCOM. (2020). Children and parents: Media use and attitudes report 2019. Retrieved from https://www. ofcom.org.uk/_data/assets/pdf_file/0023/190616/ children-media-use-attitudes-2019-report.pdf

Orben, A. (2020). Teenagers, screens and social media: a narrative review of reviews and key studies. Social Psychiatry and Psychiatric Epidemiology, 55, 407-414. https://10.1007/s00127-019-01825-4

Potter, J., \& McDougall, J. (2017). Digital media, culture \& education. Theorising third space literacies. London: Pallgrave McMillian UK.

Rivoltella, P.C. (2015). Le virtù del digitale. Per unetica dei media. Brescia: Morcelliana.

Scarcelli, C. M. (2015). Intimità digitali. Adolescenti, amore e sessualità ai tempi di internet. Milano: FrancoAngeli.

Smahel, D., Machackova, H., Mascheroni, G., Dedkova, L., Staksrud, E., Ólafsson, K., Livingstone, S., \& Hasebrink, U. (2020). EU Kids Online 2020: Survey results from 19 countries. EU Kids Online. Retrieved from https://doi.org/10.21953/lse.47fdeqj01 ofo

Sultana, A., Tasnim, S., Hossain, M. M., Bhattacharya, S., and Purohit, N. (2021). Digital screen time during the COVID-19 pandemic: a public health concern. F1000Research 2021, 10:81.

Turkle, S. (1995). Life on the Screen. New York: Simon \& Schuster.

Turkle, S. (2012). Alone Together: Why We Expect More from Technology and Less from Each Other. Basic Books. manca luogo di edizione

Twenge, J. M. (2018). Iperconnessi (Ed. Italia). Torino: Einaudi. 